\title{
Hepatocellular carcinoma in patients without cirrhosis: relevance and clinical characteristics
}

\author{
Kellyane Santana Dias Carvalho', Luciano E. Fonseca², Helma P. Cotrim ${ }^{1}$ \\ 'Medicine School, Universidade Federal da Bahia, Salvador 40110-060, Brazil. \\ ${ }^{2}$ University Hospital Prof. Edgar Santos, Universidade Federal da Bahia, Salvador 40110-060, Brazil. \\ Correspondence to: Dr. Helma P. Cotrim, Programa de Pós Graduação em Medicina e Saúde - PPgMS, Complexo Universitário Professor \\ Edgard Santos, Rua Augusto Viana, 50 Andar, Canela, Salvador 40110-060, Brazil. E-mail: helmacotrim@gmail.com \\ How to cite this article: Carvalho KSD, Fonseca LE, Cotrim HP. Hepatocellular carcinoma in patients without cirrhosis: relevance and \\ clinical characteristics. Hepatoma Res 2018;4:15. http://dx.doi.org/10.20517/2394-5079.2018.13
}

Received: 24 Feb 2018 First Decision: 21 Mar 2018 Revised: 8 May 2018 Accepted: 8 May 2018 Published: 29 May 2018

Science Editor: Guang-Wen Cao Copy Editor: Guang-Zhe Zhu Production Editor: Huan-Liang Wu

\begin{abstract}
Aim: The present study evaluated the frequency of hepatocellular carcinoma (HCC) in patients without cirrhosis.

Methods: HCC patients were recruited from two reference centers for liver disease in Northeast Brazil from 2010 to 2016. The diagnosis of HCC and cirrhosis was based on international criteria.

Results: A total of 169 patients were included, and 16\% (27) of the patients did not have hepatocellular carcinoma in non-cirrhosis (HCC-NC). The mean age of HCC-NC was $64.4 \pm 11.3$ years, and $74.1 \%$ of the patients were male. The main risk factors were hepatitis C virus (HCV) in $29.6 \%$ (8), nonalcoholic steatohepatitis (NASH) in $14.8 \%$ (4) and hepatitis B virus (HBV) in $11.1 \%$ (3). Histological HCC diagnosis was performed in $81.5 \%$ (22) of the patients, and in $18.5 \%$ (5) of these patients, the diagnosis was performed by ultrasonography, computed tomography or nuclear magnetic resonance imaging methods. Single nodules were found in 56\% of HCC-NC (14) when assessed by imaging methods.
\end{abstract}

Conclusion: The frequency of HCC-NC was elevated and more common in males. HCV, NASH and HBV were the most frequent risk factors. These data contribute to discussion on future protocols and criteria for the early diagnosis and treatment of HCC in patients with chronic liver disease without cirrhosis.

Keywords: Hepatocellular carcinoma, primary liver tumor, liver cirrhosis

\section{INTRODUCTION}

Hepatocellular carcinoma (HCC) is the most frequent primary malignant tumor found in the liver. HCC is

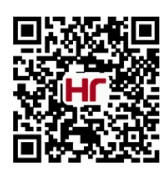


also the second cause of deaths related to cancer, accounting for 700,000 deaths every year worldwide ${ }^{[1]}$.

In Brazil, HCC is the 8th most frequent malignant neoplasm and represents approximately 10,000 cases per year $^{[2]}$.

A Brazilian national survey conducted in 2009 showed that hepatic cirrhosis was present in $98 \%$ of HCC patients, and this tumor was more frequent in cirrhosis patients with hepatitis C virus (HCV), hepatitis $\mathrm{B}$ virus (HBV) chronic hepatitis and alcoholic liver disease ${ }^{[3]}$.

However, HCC can also be associated with other liver diseases, such as non-alcoholic fatty liver disease (NAFLD), nonalcoholic steatohepatitis (NASH), and hemochromatosis as well as toxins ${ }^{[4]}$.

In patients without cirrhosis, the prevalence of HCC varies between $7 \%$ to $54 \%$ of the cases and can have a major influence on the geographical area ${ }^{[5]}$. In Western countries, the prevalence of hepatocellular carcinoma in non-cirrhosis (HCC-NC) patients was estimated in $15 \%$ to $20 \%$ of cases ${ }^{[6-8]}$, and the most common risk factors were HBV and HCV. However, a majority of the information was obtained from Asia and Africa, where the prevalence of hepatitis $\mathrm{B}$ and $\mathrm{C}$ viral infections is also elevated ${ }^{[9-11]}$.

NASH is considered a relevant risk factor of liver disease worldwide ${ }^{[12]}$. Associated metabolic syndrome manifestations may also contribute to the development of HCC in patients without cirrhosis ${ }^{[13]}$.

The present study evaluated the frequency, associated factors and clinical characteristics of HCC in Brazilian patients without cirrhosis.

\section{METHODS}

\section{Design and population study}

The present cross-sectional study included patients with HCC diagnosis from two reference centers for liver disease in Northeast Brazil from 2010 to 2016.

Inclusion criteria were as follows: patients diagnosed with hepatocellular carcinoma of different etiologies (NAFLD, HBV, HCV, alcohol, hemochromatosis, and etiology related to toxic agents)

Exclusion criteria were as follows: patients diagnosed with hepatocellular carcinoma and cirrhosis.

\section{Diagnostic criteria}

The diagnostic criteria for HCC were according to European Association for the Study of the Liver (EASL) recommendations ${ }^{[14]}$.

The criteria for the diagnosis of cirrhosis was histological and/or by the evaluation of non-invasive markers, such as FIB-4 $\left\{\right.$ FIB-4 = age $($ years $) \times$ aspartate aminotransferase $(\mathrm{AST})(\mathrm{U} / \mathrm{L}) /\left[\right.$ Platelets $(\mathrm{PLT})\left(10^{9} / \mathrm{L}\right) \times$ alanine transaminase $\left.\left.(\mathrm{ALT})^{1 / 2}(\mathrm{U} / \mathrm{L})\right]\right\}$.

\section{Clinical assessment}

All the data were obtained from a questionnaire containing the following variables: gender, age, and risk factors for liver diseases (HBV, HCV, NASH, alcohol, and metabolic- and toxic-related factors). The data from physical examinations and completed additional tests [liver, lipid, and glycemic profiles, serum insulin, hepatitis B surface antigen (HBsAg), anti-HCV, ferritin, and transferrin saturation index] were considered. All the patients were also evaluated by at least two imaging methods, such as total abdominal ultrasonography (US), computed tomography (CT) or magnetic resonance imaging (MRI). 
Table 1. Clinical characteristics of hepatocellular carcinoma patients with and without cirrhosis

\begin{tabular}{lcl}
\hline Variables & Without cirrhosis & With cirrhosis \\
\hline Gender & $20(74.1)$ & $110(77.5)$ \\
Male, $n(\%)$ & $7(25.9)$ & $32(22.5)$ \\
Female, $n$ (\%) & $64.4 \pm 11.3$ & $58.8( \pm 10.9)$ \\
Age, median \pm SD (years) & $5.3 \pm 2.9$ & $5.49( \pm 4.0)$ \\
Size, median \pm SD (cm) & $20(74)$ & $125(88)$ \\
Etiology, $n(\%)$ & $8(29.6)$ & $59(48.5)$ \\
HCV & $4(14.8)$ & $4(2.8)$ \\
NASH & $3(11.1)$ & $14(10)$ \\
HBV & $3(11.1)$ & $22(15.5)$ \\
Cryptogenic & $2(7.4)$ & $24(17)$ \\
ALD & - & $1(0.7)$ \\
Hemochromatosis & $7(26)$ & $17(12)$ \\
Risk factor unknown, $n(\%)$ & & \\
\hline
\end{tabular}

SD: standard deviation; HCV: hepatitis C virus; NASH: non-alcoholic steatohepatitis; HBV: hepatitis B virus; ALD: alcoholic liver disease

\section{Histological assessment}

Histological evaluation was performed on liver biopsies or surgical samples. The diagnostic criteria for HCC were based on the recommendations of the International Consensus Panel ${ }^{[15]}$.

\section{Statistical analysis}

The statistical analyses were descriptive and performed with the Statistical Package for the Social Sciences (SPSS) software (version 22.0, IBM Corp., USA). The data were analyzed, and the results are expressed as the mean values, standard deviations, and medians according to the distribution of the variables.

The present study was conducted according to the guidelines established in the 1964 declaration of Helsinki. The project was approved by the Research Ethics Committee at Bahia Medicine School, Federal University of Bahia, Brazil. All the participants signed letters of informed consent.

\section{RESULTS}

A total of 169 patients with HCC were evaluated, and 16\% (27) of the cases were HCC-NC. Table 1 shows the main clinical characteristics and risk factors of the patients without and with cirrhosis.

Histological analysis was performed in $81.5 \%$ of the cases $(n=22)$. A diagnosis was made by imaging methods (CT or MRI) in $18.5 \%$ of the cases [Table 2 $]^{[16]}$.

\section{DISCUSSION}

The prevalence of HCC-NC in this Brazilian study was elevated (16\%), and the results were similar to those found in other studies conducted in Western countries ${ }^{[6-8]}$. The patients were most frequently of advanced ages (mean of 64.4 years) and predominately male. These data are consistent with the findings of previous studies, although in other studies, the diagnosis of HCC-NC was more frequent in younger individuals and in women ${ }^{[5]}$. This difference may be due to the geographical variations in the prevalence of HCC and its risk factors.

Chronic HBV and HCV infections are the most frequent risk factors for HCC in patients with and without HCC-NC. An estimated $0.1 \%$ of individuals with HBV without cirrhosis develop $\mathrm{HCC}^{[9]}$, likely due to the carcinogenic effect of the virus ${ }^{[10]}$. HCV is described in most studies as being of low potential for developing HCC in the absence of cirrhosis. However, more recent studies have shown the existence of HCC-NC in patients with chronic hepatitis $\mathrm{HCV}$, suggesting that other mechanisms independent of cirrhosis would affect hepatocarcinogenesis ${ }^{[5,11]}$. 
Table 2. HCC in patients without cirrhosis from imaging methods (CT and/or MR)

\begin{tabular}{ll}
\hline Tumor numbers & Value, $\boldsymbol{n}(\%)$ \\
\hline 1 & $17(68)$ \\
2 & $3(11.1)$ \\
3 or more & $5(20)$ \\
Size, median \pm SD $(\mathrm{cm})$ & $5.1 \pm 2.7$ \\
BCLC, $n$ & \\
0 & 0 \\
A & $11(40.7)$ \\
B & $8(29.6)$ \\
C & $5(18.5)$ \\
D & 0 \\
\hline
\end{tabular}

BCLC: Barcelona Clinic Liver Cancer ${ }^{[16]} ; \mathrm{CT}$ : computed tomography; MR: magnetic resonance; SD: standard deviation

However, this scenario could change over the next few years or decades, since effective treatments for the elimination of this virus are currently being used. However, there is a growing increase in NAFLD with the prospect of becoming the leading cause of liver disease worldwide associated with risk factors, such as dyslipidemia, central obesity, diabetes and metabolic syndrome.

In the present study, HCV was also the main risk factor for HCC-NC cases, even in areas of Northeast Brazil, where the prevalence of HCV is low ${ }^{[17]}$. Perhaps, the prevalence has been influenced by the origin of the patients. The patients in the present study were recruited from reference centers for liver disease.

Chronic HBV infection was also a relevant risk factor for HCC-NC in this patient sample, even after national vaccination programs for this virus. These data are extremely concerning. HBV has a direct oncogenic effect $^{[18]}$, and patients without cirrhosis are frequently not included in protocols for the early diagnosis of this neoplasm.

$\mathrm{NASH}$, as the second most frequent risk factor after HCV, in the present series of HCC-NC patients, was observed in $14.8 \%$ of the cases. Although the prevalence of HCC without cirrhosis in patients with NASH is considered low, in some studies ${ }^{[19-21]}$, NASH also has been recognized as a relevant cause of this liver tumor in patients without cirrhosis. In addition, obesity and diabetes, the major risk factors associated with NAFLD (steatosis and NASH), are also independent risk factors for $\mathrm{HCC}^{[13,22]}$. In the present study, $33 \%$ of the HCCNC patients had diabetes.

In Brazil, a recent national survey that included 110 cases of HCC associated with NAFLD showed that 31\% of the cases, diagnosed through liver biopsy, did not present cirrhosis ${ }^{[23]}$.

In the present study, a single nodule was observed in $68 \%$ of the HCC cases. Treatment with curative intent (resection) occurred in 59.3\% of the cases. Histopathological evaluation was performed in $81.5 \%$ of the cases, and $51.9 \%$ of the HCC cases were classified as moderately differentiated tumors. This finding is interesting since the HCC diagnosis was conducted in patients without cirrhosis, who were not included in protocols for early diagnosis and treatment.

Previous studies have also shown that the majority of HCC-NC cases are diagnosed as a single and larger tumor $^{[24,25]}$, it could be explained because patients with chronic liver disease without cirrhosis are not part of the surveillance protocol, and the diagnosis was performed in patients with more advanced stages.

Although the study presents relevant data, it has some limitations. A lack of knowledge of the prevalence of HCC in the reference population is important because the frequency of HCC-NC may be underestimated. 
The majority of the clinical information was obtained from patient records, and some of the patients presented incomplete data.

In conclusion, the frequency of HCC-NC in these Brazilian patients was elevated and more commonly observed in men. HCV, NASH, and HBV were the most frequent risk factors associated with HCC-NC. These data contribute to discussions on future protocols and criteria for the early diagnosis and treatment of HCC patients with chronic liver disease without cirrhosis.

\section{DECLARATIONS}

\section{Authors' contributions}

Concept and design: Cotrim HP

Data acquisition: Carvalho KSD, Fonseca LE

Data analysis: Carvalho KSD, Cotrim HP

Manuscript preparation: Carvalho KSD, Cotrim HP

Critical revision and finalizing of the manuscript: Cotrim HP

\section{Data source and availability}

The data were strictly obtained from medical records according to the privacy policy and ethics code of our institute.

\section{Financial support and sponsorship}

None.

\section{Conflicts of interest}

There are no conflicts of interest.

\section{Patient consent}

Consents from all of the patients were established prior to submission and all records were confidential.

\section{Ethics approval}

The present study was approved by the Ethics Committee and Research at Bahia School of Medicine, Universidade Federal da Bahia, Brazil.

\section{Copyright}

(c) The Author(s) 2018.

\section{REFERENCES}

1. Ferlay J, Soerjomataram I, Ervik M, Dikshit R, Eser S, Mathers C, Rebelo M, Parkin DM, Forman D, Bray F. Cancer incidence and mortality worldwide: source, method and major patterns in GLOBOCAN 2012. Int J Cancer 2015;136:E359-86.

2. Paranaguá-Vezozzo DC, Ono SK, Alvarado-Mora MV, Farias AQ, Cunha-Silva M, França JI, Alves VA, Sherman M, Carrilho FJ. Epidemiology of HCC in Brazil: incidence and risk factors in a ten-year cohort. Ann Hepatol 2014;13:386-93.

3. Carrilho FJ, Kikuchi L, Branco F, Goncalves CS, Mattos AA; Brazilian HCC Study Group. Clinical and epidemiological aspects of hepatocellular carcinoma in Brazil. Clinics 2010;65:1285-90.

4. Fattovich G, Stroffolini T, Zagni I, Donato F. Hepatocellular carcinoma in cirrhosis: incidence and risk factors. Gastroenterology 2004;127:S35-50.

5. Trevisani F, Frigerio M, Santi V, Grignaschi A, Bernardi M. Hepatocellular carcinoma in non-cirrhotic liver: a reappraisal. Dig Liver Dis 2010;42:341-7.

6. $\quad$ El-Serag HB. Hepatocellular carcinoma. N Engl J Med 2011;365:1118-27.

7. Wörns MA, Bosslet T, Victor A, Koch S, Hoppe-Lotichius M, Heise M, Hansen T, Pitton MB, Niederle IM, Schuchmann M, Weinmann A, Düber C, Galle PR, Otto G. Prognostic factors and outcomes of patients with hepatocellular carcinoma in non-cirrhotic liver. Scand J Gastroenterol 2012;47:718-28.

8. Schütte K, Kipper M, Kahl S, Bornschein J, Götze T, Adolf D, Arend J, Seidensticker R, Lippert H, Ricke J, Malfertheiner P. Clinical characteristics and time trends in etiology of hepatocellular cancer in Germany. Digestion 2013;87:147-59. 
9. Fattovich G, Bortolotti F, Donato F. Natural history of chronic hepatitis B: special emphasis on disease progression and prognostic factors. $J$ Hepatol 2008;48:335-52.

10. Bonilla Guerrero R, Roberts LR. The role of hepatitis B virus integrations in the pathogenesis of human hepatocellular carcinoma. J Hepatol 2005;42(5):760-77.

11. Albeldawi M, Soliman M, Lopez R, Zein NN. Hepatitis C virus associated primary hepatocellular carcinoma in non-cirrhotic patients. Dig Dis Sci 2012;57:3265-70.

12. Younossi ZM, Stepanova M, Afendy M, Fang Y, Younossi Y, Srishord M. Changes in the prevalence of the most common causes of chronic liver diseases in the United States from 1988 to 2008. Clin Gastroenterol Hepatol 2011;9:524-30.

13. Welzel TM, Graubard BI, Zeuzem S, El-Serag HB, Davila JA, McGlynn KA. Metabolic syndrome increases the risk of primary liver cancer in the United States: a study in the SEER-Medicare database. Hepatology 2011;54:463-71.

14. European Association for the Study of the Liver; European Organization for Research and treatment of Cancer. EASL-EORTC clinical practice guidelines: management of hepatocellular carcinoma. $J$ Hepatol 2012;56:908-43.

15. International Consensus Group for Hepatocellular Neoplasia. Pathologic diagnosis of early hepatocellular carcinoma: a report of the international consensus group for hepatocellular neoplasia. Hepatology 2009;49:658-64

16. Llovet JM, Burroughs A, Bruix J. Hepatocellularcarcinoma. Lancet 2003;362:1907-17.

17. Pereira LM, Martelli CM, Moreira RC, Merchan-Hamman E, Stein AT, Cardoso MR, Figueiredo GM, Montarroyos UR, Braga C, Turchi MD, Coral G, Crespo D, Lima ML, Alencar LC, Costa M, Dos Santos AA, Ximenes RA. Prevalence and risk factors of hepatitis C infectious in Brazil. 2005 through 2009: a cross-sectonal study. BMC Infect Dis 2013;13:60.

18. Farazi PA, Depinho RA. Hepatocellular carcinoma pathogenesis: from genes to environment. Nat Rev Cancer 2006;6:674-87.

19. Adams LA, Lymp JF, Sauver J, Sanderson SO, Lindor KD, Feldstein A, Angulo P. The natural history of nonalcoholic fatty liver disease: a population-based cohort study. Gastroenterology 2005;129:113-21.

20. Ekstedt M, Franzén LE, Mathiesen UL, Thorelius L, Holmqvist M, Bodemar G, Kechagias S. Long-term follow-up of patients with NAFLD and elevated liver enzymes. Hepatology 2006;44:865-73.

21. Dam-Larsen S, Franzmann M, Andersen IB, Christoffersen P, Jensen LB, Sørensen TI, Becker U, Bendtsen F. Long term prognosis of fatty liver: risk of chronic liver disease and death. Gut 2004;53:750-5.

22. Rosmorduc O, Fartoux L. HCC and NASH: how strong is the clinical demonstration? Clin Res Hepatol Gastroenterol 2012;36:202-8.

23. Cotrim HP, Oliveira CP, Coelho HS, Alvares-da-Silva MR, Nabuco L, Parise ER, Ivantes C, Martinelli AL, Galizzi-Fiho J, Carriho FJ. Members of the Brazilian society of hepatology HCC. Clinics (Sao Paulo) 2016;71:281-4.

24. Alkofer B, Lepennec V, Chiche L. Hepatocellular cancer in the non-cirrhotic liver. J Visc Surg 2011;148:3-11.

25. Sarpel U, Ayo D, Lobach I, Xu R, Newman E. Inverse relationship between cirrhosis and massive tumours in hepatocellular carcinoma. HPB (Oxford) 2012;14:741-5. 